\title{
Essais
}

Revue interdisciplinaire d'Humanités

Narration et lien social

\section{Gherardo Ortalli, Barattieri. Il gioco d'azzardo fra economia ed etica. Secoli XIII-XV}

\section{Marco Conti}

\section{(2) OpenEdition}

1 Journals

\section{Édition électronique}

URL : https://journals.openedition.org/essais/9565

DOI : $10.4000 /$ essais. 9565

ISSN : 2276-0970

Éditeur

École doctorale Montaigne Humanités

\section{Édition imprimée}

Date de publication : 15 mai 2013

Pagination : 215-217

ISBN : 978-2-9544269-0-7

ISSN : 2417-4211

\section{Référence électronique}

Marco Conti, «Gherardo Ortalli, Barattieri. II gioco d'azzardo fra economia ed etica. Secoli XIII-XV », Essais [En ligne], 3 | 2013, mis en ligne le 01 septembre 2021, consulté le 08 septembre 2021. URL : http:// journals.openedition.org/essais/9565; DOI : https://doi.org/10.4000/essais.9565 


\section{Comptes rendus}

Gherardo Ortalli, Barattieri. Il gioco d'azzardo fra economia ed etica. Secoli XIII-XV, Bologne, Mulino, 2012.

Le terme barattiere est actuellement utilisé dans la langue italienne pour désigner celui qui, en charge d'un office public, a été corrompu. Cette définition correspond aussi à une des acceptions qui était retenue au Moyen Age. Dante Alighieri, dans la Commedia, fait référence à la baratteria car lui-même fut accusé et successivement condamné en 1302 pour ce crime. Mais, plus usuellement, le terme baratteria était associé à la notion ambiguë de marginalité, et au monde de ces mali homines qui pratiquaient les jeux de hasard. C'est surtout cette dernière idée qui fait l'intérêt de la recherche de Barattieri. Il gioco d'azzardo fra economia ed etica, qui enquête sur le jeu de hasard dans les villes de l'Italie communale. Gherardo Ortalli professeur d'Histoire Médiévale à l'Université Ca' Foscari de Venise est l'auteur de nombreuses recherches de haut niveau, et notamment de La peinture infamante du XIII au XVI siècle. ${ }^{1}$. Cela fait plusieurs années qu'il s'intéresse au monde du ludique, et ce dernier livre est une étape importante de ce parcours, ou pour mieux dire, un premier point de départ pour ce type de recherche. Barattieri. Il gioco d'azzardo fra economia ed etica a été publié en octobre 2012 pour Il Mulino, et il est structuré en cinq chapitres pour un total de 264 pages.

Le premier chapitre présente le panorama historiographique sur la thématique du ludique, laquelle est plutôt pauvre d'ouvrages, avant de nous montrer les relations entre culture économique, jeu de hasard, loi et morale. La société médiévale ne voyait pas dans le jeu en lui-même un risque pour la stabilité de la société, en revanche, le jeu lié au hasard était mal considéré, car cette

1 G. Ortalli, La peinture infamante du XIII a au XVIe siècle, Paris, 1994 ; traduit de l'italien par Fabienne Pasquet et Daniel Arasse, (éd. originale Pingatur in palatio. La pittura infamante nei secoli XIII-XVI, Rome, 1979). 
notion de hasard était assimilée à ce monde fait d'infamies, de blasphèmes, et de ces hommes prêts à tout jouer jusqu' à leur dernière chemise. Les villes commencèrent à légiférer sur ce phénomène, pour bien l'encadrer, pour éviter les désordres ainsi que pour utiliser en leur faveur des concessions à travers des taxations sur le jeu. Car le jeu devenait un nouveau type de revenus pour la ville, qui durant cette période commençait à faire front aux premiers symptômes de la dette publique. Le jeu de hasard va ainsi trouver une certaine légitimité au travers de la gabella baraterie. Cependant, il faut analyser cette évolution comme un compromis (et non comme une véritable acceptation de cette pratique), dans la mesure où, de temps en temps, les villes interdisaient ces pratiques, ou les limitaient, pour les reprendre quelques années plus tard (comme ce fut le cas à Bologne).

Le second chapitre analyse les groupes sociaux et les structures liés à la baratteria. Dans les sources relatives à la ville de San Gimignano, se retrouve la première attestation, précisément en 1250, du mot baratteria utilisé pour désigner le lieu où était autorisé, et presque officialisé, le jeu de hasard (notamment et surtout le jeu de dés). Ces lieux étaient administrés par des fermiers des impôts, lesquels, pour avoir la gestion de cette taxe indirecte, participaient à des adjudications publiques. Dans ce chapitre, Ortalli montre admirablement les contradictions relatives à ces charges publiques impliquées dans un monde perçu comme ambigu. La ville constituait des listes où étaient enregistrés tous les joueurs de hasard, dans le but d'avoir le maximum de contrôle sur cette partie de la société. Tous ces facteurs donnent au jeu de hasard une légitimation publique.

Barattieri est le titre du troisième chapitre, qui est destiné à étudier cette " catégorie » de joueurs de hasard et les rapports entre ceux-ci et le gouvernement des communes. La perception de ces gens par la société reste incertaine. Ces hommes étaient souvent appelés par la ville pour l'exécution de tâches mal considérées, pour exercer par exemple les fonctions de bourreau. La torture était admise à leur encontre, ainsi que leur condamnation à toutes les peines liées au mépris. À l'inverse, les personnes qui géraient l'encaissement de la taxe sur les jeux, étaient des individus respectés par la société, qui, en possédant déjà des fortunes modestes, avaient la possibilité d'acquérir la gestion du dacium ludis à travers les adjudications publiques. Enfin, l'auteur veut faire la lumière sur l'organisation interne de ce système, avec un croisement de sources, pour bien comprendre l'utilisation des termes comme rex baratterie ou societas.

Le quatrième chapitre est dédié à l'adjudication faite par la ville sur le dacium ludis, et l'importance économique de cette taxation. Même si elle n'était pas un des principaux revenus des caisses de la ville, elle était importante dans la recherche chronique de nouveaux financements pour les bilans communaux. Ces taxations pouvaient être aussi directement destinées à des financements des travaux publics, ou à d'autres dépenses ad hoc. 
Enfin, le dernier chapitre analyse les mutations que ce système a connues durant le $\mathrm{XV}^{e}$ siècle et au cours duquel les rapports de force entre éthique et économie changèrent. Le climat en matière de jeu public est modifié. Les hostilités et les condamnations demeurent mais dans un contexte nouveau dans lequel il faut nier les choix pris dans le passé, et les montrer à posteriori comme des malentendus. Ce jeu de force s'est achevé en faveur de la morale grâce au grand travail entrepris par les ordres des prédicateurs, faisant des sermons en public et en brûlant des dés, des cartes, etc. Il n'y avait plus de place pour le jeu de hasard dans les lieux publics, ce qui eut pour conséquence d'une part le déplacement du jeu de hasard dans des maisons privées dans lesquelles il se pratiquait désormais loin des regards de la loi, et d'autre part, le développement de nouvelles pratiques institutionnalisées comme la loterie.

Dans cet ouvrage important, Gherardo Ortalli a décidé d'observer des aspects qui sont considérés comme marginaux ou négligeables dans d'autres recherches. C'est surtout cette approche, réalisée avec un croisement de sources de différentes natures (économique, judicaire, morale, religieuse, etc.), qui a donné ce remarquable tableau de la société médiévale italienne. Cette stratégie de travail a permis d'identifier toutes les contradictions de ces pratiques du jeu de hasard, ainsi que l'équilibre entre moralité et pratique économique qui était très précaire. La même analyse de l'évolution du terme barattiere démontre bien cette situation oscillant toujours entre illégalité et légalité. À noter que si l'ouvrage examine la situation des villes du centre-septentrional de l'Italie, il ne manque pas de références à l'Europe du Nord, à l'Espagne, à la France, et à d'autres pays européens, où, vers la fin du XIII siècle, la baratteria vient à se structurer comme une entreprise financière, et où le jeu de hasard est géré au profit des finances de la ville, par des hommes d'affaires et de finance. Si dans un premier temps l'intérêt économique a pris le pas sur la morale, dans en second temps, c'est la morale qui oblige les villes à adopter de nouvelles lois, de nouvelles prohibitions, et à créer de nouveaux systèmes pour assurer ces revenus. C'est dans ce contexte que le mot barattiere correspond à des valeurs plus génériques qu'avant, et précisément dans son acception moderne. La rigueur et la méthodologie de recherche fait du livre de Gherardo Ortalli une indiscutable référence, tant pour les recherches en histoire médiévale du ludique que pour d'autres recherches qui peuvent prendre à cet auteur sa magistrale façon de travailler.

Marco Conti

Master 1 Recherche Italien Université Michel de Montaigne Bordeaux 3 mrc.conti87@gmail.com 